This paper is a preprint of a paper submitted to and accepted for publication in IET Communications and is subject to Institution of Engineering and Technology Copyright.

The copy of record is available at IET Digital Library.

Some final publisher edits and corrections may not be included in this file.

\title{
Multi-tone Frequency Shift Keying for Ultrawideband Wireless Communications
}

Vit Sipal ${ }^{1}$, Ben Allen ${ }^{2}$, David Edwards ${ }^{1}$

${ }^{1}$ Dept. of Engineering Science University of Oxford, Parks Road, Oxford OX1 3PJ

${ }^{2}$ Centre for Wireless Research, University of Bedfordshire, Park Square, Luton LU1 3JU

\section{Abstract}

This paper explores the performance of Multi-tone Frequency Shift Keying (MT-FSK) in ultrawideband wireless communications as an alternative to the standard modulation techniques. Its advantage is its ability to provide multiple user access even within the recently tightened regulations on UWB communications. Unlike previous works that discussed MT-FSK from the information theory perspective, here the possibility of practical implementation is explored. The basic system parameters such as tone bandwidth, number of frequencies and duty cycle are studied in terms of propagation characteristics, optimum data rate and system complexity. With optimum parameters, we show that a system using Multi-tone Frequency Shift Keying is capable of data transmission at a data-rate of $250 \mathrm{Mbps}$ with Bit-Error-Rate below $10^{-5}$ whilst the receiver complexity is lower than for standard methods utilising the ultrawideband spectrum by means of impulse radio or multi-band OFDM.

\section{Introduction}

The interest in Ultrawideband (UWB) technology as a means of high data rate short range wireless communication started in the late 1990s after Scholtz et.al. showed that it is possible to avoid catastrophic collision of multiple users for baseband impulse radio [1]. The interest of industry and academia was amplified in 2002 after the US FCC had approved the $3.1-10.6 \mathrm{GHz}$ spectrum for UWB communication. After this ruling, various modulation schemes were investigated. 
Over the following ten years two approaches crystallised as main stream - the Multiband OFDM approach supported mainly by the WiMedia alliance [2], and the Impulse Radio approach that remains in the focus of many research groups worldwide. Unfortunately, neither of these approaches has fulfilled the initial expectations associated with UWB in terms of a provider of lowcost gigabit wireless.

For Impulse Radio, the guard intervals between pulses need to be sufficient to avoid inter-symbol interference. As a result, the transmitter operates at a very low duty cycle and achieving data-rates in the magnitude of hundreds of Mbps does not seem possible. The future of impulse radio seems to be in low cost low data-rate applications such as wireless sensor networks.

For a significant period of time, the Multiband OFDM approach seemed to be the preferred choice for UWB by both academics and the industry [2]. Initially, its main issue was considered the chip complexity associated with the UWB implementation (compared to the FFT implementation for the OFDM in 802.11, the UWB's FFT implementation is approx. 20 times more complex) [3]. However, it was only seen as a hurdle that will eventually be overcome. Unfortunately, the new regulatory developments have put the WiMedia systems in a difficult situation. In some of the major markets, the regulatory limits imposed on UWB communication have changed significantly. For the USA, the UWB band remains $3.1-10.6 \mathrm{GHz}$. In Europe, the UWB band has recently been limited only to 6 8.5 GHz [3]. Additionally, the $3.1-4.8 \mathrm{GHz}$ and $8.5-9 \mathrm{GHz}$ bands can be used when Detect-andAvoid Algorithms (DAA) are implemented [3]. In Japan, the UWB band is now limited to $7.25-10.25$ $\mathrm{GHz}$ without DAA and $3.4-4.8 \mathrm{GHz}$ band is possible with DAA [3]. A similar situation prevails in Korea, where the UWB without DAA is allowed in the $7.2-10.2 \mathrm{GHz}$ range and DAA enables an additional band between 3.1 and $4.8 \mathrm{GHz}$.

The impact of these regulatory changes on WiMedia UWB systems is enormous. WiMedia employs frequency hopping over three bands - thus each user occupies $1.584 \mathrm{GHz}(3 \times 528 \mathrm{MHz})$ [2]. In terms of multiple user access, the specification relies on frequency division multiple access. This effectively means that for chips without DAA co-existence of two users is impossible. With DAA, a second user 
can be accommodated in Europe and in Korea but not in Japan where the additional DAA band provides only $1.4 \mathrm{GHz}$ bandwidth. A simple change of the hopping schemes is also not very straightforward as the hopping was designed specifically to spread the information over multiple carrier frequencies in order to combat frequency selective fading of the channel [2]. Hence, a change in the band hopping scheme also requires a complete re-design of channel coding.

Therefore, there is strong motivation to develop schemes that can accommodate multiple users within the stricter regulatory limits - ideally within $2.5 \mathrm{GHz}$ so that the system is transferable into all major markets without the need to employ DAA. In this work, we consider and evaluate Multiple Tone Frequency Shift Keying as a potential candidate.

The standard single-tone FSK has been considered for UWB wireless communication in [4], where it is used for a low complexity transceiver achieving bit rates of 1-15 Mbps. Compared to single-tone FSK, multitone FSK, introduced in [5] and [6], transmits more tones at a time, thus bit blocks are represented by the combination of the frequencies. Due to the use of the combinations, the size of the alphabet is increased, which increases the bit-rate and the spectral efficiency of the scheme whilst the receiver complexity remains lower than in the case of MB-OFDM, because the complexity is often quoted as one of the main challenges for MB-OFDM [3],[7].

As far as the authors are aware, authors of references [5], [6] are the only ones who have considered multitone FSK for wideband channels. Their works, however, deal with information theoretical aspects of multitone FSK (MT-FSK) and its ability to approach wideband channel capacity. This paper, in contrast, focuses on the optimisation of the scheme based on the state-of-the-art knowledge of wave propagation and the issues of practical implementation of the scheme and its performance over real channels is discussed. The paper is structured as follows. Firstly, the MT-FSK is briefly introduced. This is followed by a theoretical discussion of the impact of the channel impulse response on the performance of communication signals with arbitrary bandwidth. The impact of frequency selective fading on narrowband signals as well as the risk of inter-symbol-interference for short pulses is discussed and optimum signal bandwidths balancing both issues is proposed. These 
conclusions are confirmed by the empirical study of performance of MT-FSK systems over real indoor channels. Finally, the optimum design for MT-FSK UWB systems is proposed and its performance is thoroughly evaluated.

\section{Multitone FSK}

The block diagram of a transmitter and a receiver using energy detectors is plotted in Fig. 1 . The transmitter selects $K$ out of $N$ tones, each set to a different centre frequency. The set of $K$ tones represents a block of $M$ bits. The receiver can be realised as a filter bank and a power integrator behind each filter is used to determine the presence of a certain frequency. A selection algorithm picks the $K$ most likely frequencies and recovers $M$ bits.

One of the advantages of the scheme, apart from the low complexity, is the possibility of accommodating multiple users by the means of code division. I.e. different users are assigned different tone combinations and invalid code combinations are rejected by the receiver. This approach can also be used to improve the spectral efficiency. If a transmitter uses two orthogonal sets of codes then it can reduce the guard interval between subsequent symbols by alternating the code set used.

There are many factors influencing the choice of the number of tones, $\mathrm{N}$, and the number of active tones, $\mathrm{K}$. One of them is the consideration of the symbol-error-rate (SER), which can be expressed as follows:

$S E R=1-\left[\left(1-P_{e 1}\right)^{N-K}\right]^{K}=1-\left(1-P_{e 1}\right)^{N K-K^{2}}$

$P_{e 1}$ is a probability that one of the $(\mathrm{N}-\mathrm{K})$ detectors whose tones are not active yields a higher output than the active channel and it will be selected instead.

The term $\left(1-P_{e 1}\right)$ expresses the probability that one of the $K$ active tones will be selected over one of the $(N-K)$ non-selected tones. As the tones are assumed to be independent since, for sufficient spacing, the channels manifest frequency diversity for each tone, this has to occur independently for 
all $(N-K)$ non-selected tones, thus $\left(1-P_{e 1}\right)^{N-K}$. Furthermore, the same probability must happen independently for all $\mathrm{K}$ active tones, hence $\left[\left(1-P_{e 1}\right)^{N-K}\right]^{K}$ for the probability that the symbol will be transmitted correctly.

Equation (1) gives insight into the selection of the parameter $K$ and $N$. Assuming $\mathrm{K}$ can be any number from $1-(N-1), S E R$ is symmetric around $K=N / 2$ and it is minimised for low $K=1$ or $K=N-1$. As a result, the choice of low $K$ is vital. On the other hand, $K>1$ increases the alphabet so more bits-per-symbol can be transmitted. Hence $K$ should be kept low yet a compromise between the $S E R$ and data-rate has to be considered. The symmetric alternative of $\mathrm{K}$ close to $(K-1)$ is not considered as such a system would have the same performance but more pulses would require more energy for transmission.

In terms of the number of tones, $N$, (or tone bandwidth) from (1), it can be seen that lower $N$ (larger bandwidth) is desirable as it reduces the total $S E R$.

Further selection of the number of frequencies, $\mathrm{N}$, will be given by the propagation properties. The design complexity and by the regulatory limits. The propagation properties are also crucial for the selection of pulse duty cycle. The optimisation of these properties is discussed in section 3 from the theoretical point of view and in section 4 based on simulations in the real ultrawideband channel.

The bit block size, $M$, and number of selected tones, $K$, will be given by the number of centre frequencies, $N$, required data rate and also multiple user access requirements. Choice of these parameters will be discussed in sections 3-5.

\section{Impact of Channel Impulse Response on Signal Transmission}

In this section we will discuss the impact of the channel impulse response on wireless communication. The modulation assumed is ON-OFF keying and energy detector is employed, which is in accordance with the discussed MT-FSK and the proposed detection. Whilst this assumption is specific and suitable for the studied MT-FSK modulation, the conclusions on bandwidth selection considering the channel impulse response remain transferrable to other modulation schemes as well. 
In an indoor environment, one of the main characteristics of the channel is the multipath propagation and it represents one of the main channel features considered in system design. The standard channel impulse response model used for UWB is summarised in the IEEE 802.15.4a model [8]:

$h(\tau)=\sum_{l=1}^{L_{c}} \sum_{k=1}^{L_{r}} \alpha_{k, l} e^{j \varphi_{k, l}} \delta\left(\tau-T_{l}-\tau_{k, l}\right)$

where $h(\tau)$ is the channel impulse response, $\alpha_{k, l}$ and $\varphi_{k, l}$ represent the multipath gain and phase coefficient of the $k$ th ray in $l$ th cluster, $T_{l}$ stands for the time-of-arrival of $l$ th cluster, $\tau_{k, l}$ is ray arrival time within individual cluster, $\delta(t)$ is Dirac impulse.

\subsection{Frequency Selective Fading}

Frequency selective fading is caused by the fact that multiple signal copies, representing multipaths, arrive at the receiver. For signals longer than the average delay between multipaths there is a superposition of the signal that might amplify or attenuate the received signal energy. For wideband short pulses with duration shorter than the delay between multipaths, the receiver can resolve individual multipaths in the time domain and the received energy is given by the total energy of the channel impulse response. Frequency selective fading has been extensively studied e.g. in. [9-11].

Using measurements from [12], Fig. 2 illustrates the impact of bandwidth on fading. The measurement was performed in an indoor laboratory environment. A channel transfer function was measured for 1,600 measurement positions. The channel energy was determined for each point for four bandwidths $(4,40,150$ and $1,000 \mathrm{MHz})$. Using these channel energies, the relative fading in $\mathrm{dB}$ was evaluated for each data point and used to plot the empirical cumulative distribution function. A centre frequency of $7.25 \mathrm{GHz}$ was chosen as it is the centre frequency of the EU UWB band 6 8.5 GHz. It can be observed that, whilst the energy of a narrowband signal might vary by a factor of up to $20 \mathrm{~dB}$, there is almost no variation in energy for the wideband signals.

The impact of the empirical cumulative distribution function on a transmission of a single signal with varying bandwidth is presented in Fig. 3, which shows the probability that a received signal will below the sensitivity level of the receiver. This probability is calculated as follows. UWB transmission 
at an equivalent isotropic radiation power (EIRP) spectral density of $-41.3 \mathrm{dBm} / \mathrm{MHz}$ is assumed and combined with the mean path loss calculated using IEEE802.15.4a channel model [8] for office environment with a path loss coefficient of 1.63 and path loss at a distance of $1 \mathrm{~m}, P L_{0}$, which equals to $36.6 \mathrm{~dB}$. This is compared to the sensitivity of the receiver. As mentioned above, ON-OFF Keying with energy detection is assumed. A state-of-the-art energy detector is reported to provide sensitivity of $-97 \mathrm{dBm} / \mathrm{MHz}$ [14]. Using results from Fig. 1, it the probability that the signal's fading will be lower than the margin between received power and receivers sensitivity. As can be seen, this probability is significantly higher for narrowband systems.

To evaluate the effect of bandwidth on the performance of wireless communication, the results from [11] were used to evaluate the BER of communication using on-off keying. The closed form expression for the BER is given as [13]:

$B E R=\frac{1}{2} \operatorname{erfc}\left(\sqrt{\frac{E_{\mathrm{b}}}{4 N_{0}}}\right)$

The actual $\frac{E_{b}}{N_{0}}$ varies significantly due to the impact of fading. As shown in Fig. 1, the variation can be up to $20 \mathrm{~dB}$ for the case of narrowband communication. Therefore, (1) can be adopted to include the statistics of fading:

$B E R=\int_{0}^{\infty} \frac{1}{2} \operatorname{erfc}\left(\sqrt{\frac{F}{4} \mathrm{E}\left\{\frac{E_{\mathrm{b}}}{N_{0}}\right\}}\right) \mathrm{p}(F) \mathrm{d} F$

Where $F$ represents the fading and is a positive random variable with probability distribution function $\mathrm{p}(F)$. $\mathrm{E}\left\{\frac{E_{\mathrm{b}}}{N_{0}}\right\}$ is the mean signal-to-noise ratio given by path-loss without considering the impact of fading. In our previous work in [11], we showed that $\mathrm{p}(F)$ is Nakagami distributed and we explored the dependency of the Nakagami parameters on bandwidth. Here, the fading parameters from [11] were used to determine the $B E R$ for $E\left\{\frac{E_{\mathrm{b}}}{N_{0}}\right\}=10 \mathrm{~dB}$ for signals with varying bandwidth. The results are plotted in Fig. 4.

This effect is well known and it might be objected that narrowband frequency selective fading is of no interest for UWB systems. Whilst UWB systems posses bandwidths beyond $500 \mathrm{MHz}$, for OFDM 
systems the narrowband fading impacts individual narrowband subcarriers. Spreading of information bits over multiple subcarriers as well as forward error correction can mitigate the impact of narrowband fading on OFDM UWB symbols [2], but it represents additional increase in computational power and reduction of spectral efficiency. On the other hand, the advantage of OFDM systems is in high duty cycle, as will be discussed in subsection 3.2.

\subsection{Inter-symbol Interference}

Whilst short wideband signals are not affected by the frequency selective fading (see Fig. 2) they are exposed to the risk associated with inter-symbol-interference. Therefore, a guard interval must be employed to avoid inter-symbol interference. Typically, the guard interval is chosen according to the delay spread of the channel. However, the measurements for IEEE 802.15.4a channel model were performed with a significantly higher dynamic range than available to practical UWB systems. Hence, we will use the IEEE802.15.4a channel model for an office environment to estimate the required guard interval considering the limited sensitivity of the a state-of-the-art energy detector, $-97 \mathrm{dBm} / \mathrm{MHz}$ [14]. This corresponds to a dynamic range of approx. $56 \mathrm{~dB}$, assuming an EIRP spectral density of $-41.3 \mathrm{dBm} / \mathrm{MHz}$.

For our estimation, we assume a signal centre frequency of $7.25 \mathrm{GHz}$ (centre of the EU UWB band). When the gain of transmitter and receiver antenna is assumed to be $1.5 \mathrm{dBi}$ for each antenna, the path loss for the direct path in the distance of $1 \mathrm{~m}$, can be calculated using the Friis formula for free space propagation [15] which yields $46.6 \mathrm{~dB}$.

The IEEE 802.15.4a channel model defines exponential decay of the energy within a cluster using coefficient $\gamma_{0}=6.4 \mathrm{~ns}$. According to this, rays arriving with a delay of $40 \mathrm{~ns}$ are expected to be exposed to attenuation of a further $26.7 \mathrm{~dB}$ (this excludes further attenuation associated with reflection losses). As a result, the received signal will be below the sensitivity of the energy detector by more than $10 \mathrm{~dB}$. A practical conclusion is that, for wideband signals shorter than $40 \mathrm{~ns}$, a guard interval will be required to protect the communication from the impact of inter-symbol-interference. 
This significantly reduces the data-rate. For impulse radios with bandwidths above $500 \mathrm{MHz}$, the maximum achievable data-rate is then bound by $25 \mathrm{Mbps}$ given by the symbol repetition rate.

\subsection{Bandwidth optimisation for UWB signal transmission}

Sections 3.1 and 3.2 presented two issues associated with transmission of UWB signals. Narrowband subcarriers as used in OFDM systems suffer from frequency selective fading. Therefore, they have inferior BER performance compared to impulse radio systems. On the other hand, impulse radio systems suffer from low spectral efficiency due to the inclusions of guards intervals. To evaluate these differences we propose following performance measure $P$ :

$P=\frac{B E R}{\left(\frac{1}{B} /\left(\frac{1}{B}+G\right)\right)^{2}}$

where the denominator represent the duty cycle of the transmission. $B$ stands for bandwidth, $\frac{1}{B}$ is an estimate for symbol duration, $G$ is the guard interval. For wideband signals shorter than $40 \mathrm{~ns}, G$ is chosen to ensure repetition rate of $40 \mathrm{~ns}$. For symbols longer than $40 \mathrm{~ns}, G=0$.

This performance parameter weighs the $B E R$ performance with the spectral efficiency of the system. The duty cycle varies from 0.0062 for $4 \mathrm{GHz}$ to 1 for the narrowband channel. The square of the duty-cycle is chosen in (4) because it gives a similar range to the change of the $B E R$ (Fig. 4) so that both effects are weighted as equally important. In terms of meaning, the measure should be minimised as minimisation $P$ of corresponds to high duty cycle and low BER. The results are plotted in Fig. 5.

\subsection{Bandwidth optimisation conclusions}

The performance measure introduced in Section 3.3 achieves optimum value for bandwidth in the interval between $150 \mathrm{MHz}$ to $550 \mathrm{MHz}$. This bandwidth interval corresponds to a compromise between the two traditional approaches towards utilisation of UWB spectra - the OFDM approach that relies on many narrowband subcarriers transmitted in parallel and the impulse radio approach that uses extremely short pulses. Indeed, the system has the benefits of both approaches. For these bandwidth, the deterioration in spectral efficiency is not as significant as in the case of much wider 
bandwidths. Impulse radios whereas the system already benefits from the ability to resolve individual multipath components in time domain so that the communication is not disrupted by frequency selective fading.

So far, the discussion has focused on optimisation of bandwidth of one signal. Due to the regulatory requirements, signals with bandwidth below $500 \mathrm{MHz}$ do not qualify as UWB transmission. However, since the signals with this bandwidth combine the advantages of OFDM and IR signals, both approaches can also be combined in implementation. It is possible to satisfy the regulatory criteria whilst capitalising on the advantages outlined above. MT-FSK investigated in this paper is an attractive option as it can operate using individual tones with bandwidths from the optimum range whilst satisfying the UWB requirements.

Whilst the conclusions here were for ON-OFF keying and the use of energy detection only, they are also applicable to other modulation schemes. The frequency selective fading has a similar impact on SNR for any modulation scheme. In terms of duty cycle, the guard interval estimation needs to be adopted based on the actual sensitivity of chosen detector, but it remains that the guard interval needs to increase as the signals become shorter in the time-domain.

\section{Simulation}

For the initial modelling, the following system was used. The number of frequency bins, $N$, was selected to be 10. This was selected as a compromise between low $N$ as concluded in section 2 and a large alphabet required for high data rates. The number of selected tones, $K$, was selected to be 4 as a compromise between low $K$ and good data rate; the resulting size of the alphabet is therefore 210 , thus the number of bits-per-block was $M=7$. The assignment of the bit blocks to frequency sets was straight forward - the first 128 combinations were chosen. Whilst, this is a very simple approach, it is believed to be sufficient for the initial tone bandwidth optimisation.

\subsection{Impact of tone bandwidth}

To investigate the effect of bandwidth, bandwidths were from interval the $5-3,000 \mathrm{MHz}$, spaced logarithmically. The centre frequencies were chosen so that the centre frequency in for the entire 
system is at $7.25 \mathrm{GHz}$. The pulse shape was selected to be a Gaussian pulse modulated to the given frequency. Its generation in practice is convenient, and also it reduces the intersymbol interference. For bandwidths above $800 \mathrm{MHz}$, the number of centre frequencies was reduced so that the total bandwidth would not exceed the bandwidth available by the measurement bandwidth, the number of selected tones as well as the bits per block were selected accordingly.

The duty cycle for the initial optimisation was selected to be extremely low (1 ms guard interval between pulses) so that no inter-symbol interference can occur.

The channel measurements used for the system simulation were the same that as reported in [12]. The laboratory where the measurement was conducted is shown in Fig. 6. The distance between transmitter and receiver was selected to be approx. 3 meters. The transmitter was moved in a grid of 40 by 40 points spaced by $6 \mathrm{~mm}$. The frequency range was $3-20 \mathrm{GHz}$, the employed antennas were discone antennas with a lower cut-off frequency of $2.5 \mathrm{GHz}$.

The simulation was conducted as follows. Firstly, the receiver is calibrated for transmitter position $[1,1]$ in the grid. The receiver first uses a calibration with a pilot sequence to estimate the channel parameters. The pilot sequence consists of all tones and the associated energy levels, $E_{i, p i l o t}$, at the receiver are determined for each finger as well as the relative timing of the decision. The receiver then determines the energy levels, $E_{i, j}$, at the output of each finger at times given by the pulse repetition rate and the timing determined during the initial equalisation. Note that $j$ stands for $j$-th symbol. These energy levels are compared to the equalisation energy levels, $E_{i, p i l o t}$. A soft decision is applied by selecting the $\mathrm{K}$ fingers with the maximum relative value of $E_{i, j} / E_{i, p i l o t}$. This allows to take into account the frequency selectivity of path loss.

$2^{20}$ randomly generated (equal probability of 0 and 1 ) bits were modulated and sent over the channel and BER was determined. This was repeated four times for different channel transfer functions representing different transmitter locations. The initial position of transmitter was in corner of the grid $[1,1]$.Subsequent simulations with chosen channels shifted diagonally by $3.6 \mathrm{~cm}$ in $x$ - and $y$ - direction. Thus, the subsequent measurement positions were $[7,7],[13,13],[19,19]$ and 
$[25,25]$. The maximum displacement was $14.4 \mathrm{~cm}$ in both $\mathrm{x}$ - and $\mathrm{y}$-directions. For these positions, still the calibration for the initial position of $[1,1]$ was used. This should simulate a potential movement that the receiver or transmitter may undergo within the interval between consequent pilot calibrations. Calibration has to be performed repeatedly but with a very low repetition period. For instance, if the repetition rate is $10 \mathrm{~ms}, 14.4 \mathrm{~cm}$ displacement within $10 \mathrm{~ms}$ corresponds to the velocity of $14.4 \mathrm{~m} / \mathrm{s}(51.8 \mathrm{~km} / \mathrm{h})$, which is extremely fast for a human in an indoor environment. Fig. 7 presents the mean relative BER for all five positions as a function of bandwidth.

As can be seen in Fig. 6, mean BER is high for high and low bandwidths corresponding. For narrowband signals bandwidths, fading of narrowband channels occurs and is more severe as illustrated in Fig. 2. Additionally, the pulses use constant power spectral density. Thus for narrowband pulses, the signal-to-noise ratio is inherently lower. The BER increase is significant for narrowband channels and is present for the bandwidths below $100 \mathrm{MHz}$. The result of this empirical study therefore well matches the theoretical predictions from Section 3.

For wideband signals, the increase in BER is associated due to the fact that the pulse length becomes short. Thus, even for a relatively small displacement the change of delay is significant compared to the length of the pulse. This is shown in more detail in Fig. 8 presenting the BER for each measurement position in terms of displacement in the diagonal direction. For each bandwidth, there is a dashed line representing the $3 \mathrm{~dB}$ duration of the Gaussian pulse in the time domain multiplied by the speed of light to correspond to the spatial displacement. It can be seen that once the displacement is approximately over the $3 \mathrm{~dB}$ run length the BER increases rapidly. Thus, the estimation of time-of-arrival for the initial position does not give the correct delay and an increase in BER occurs.

For low bandwidths Fig. 7 gives similar results as the performance measure from preceding chapter, for large bandwidths it points out additional issue of impulse radio - impulse radio with very short pulses tends requires equalisation more often as even a small spatial displacement can negatively impact the timing of the receiver. 


\subsection{Duty cycle selection}

The duty cycle of the transmit waveform must be selected based on the delay spread of the wireless channel in order to minimise inter-symbol interference. The theoretical discussion in Section 3 concludes that pulse repetition period of 40 ns should provide. Here we repeat the simulations from section 4.1 for signals with $150 \mathrm{MHz}$ bandwidth and variable symbol repetition rate in order to explore the impact of repetition rate on the BER performance.

This is illustrated in Fig. 6 which shows the BER for a transmitter in position $[10,10](8.5 \mathrm{~cm}$ from calibrated position) with a bandwidth of $150 \mathrm{MHz}$ for different symbol repetition periods. It can be seen that above $30 \mathrm{~ns}$ the BER is approaching very low values. For repetition rates below the signal duration (overlapping of tones), the BER approaches the maximum possible value of 0.5 . Based on the results from Fig. 9, it appears that the theoretical value of 40 ns derived in section 3 should provide sufficient guard interval to avoid inter-symbol-interference.

\section{Performance evaluation}

The results from section 4 were taken into account and an optimum MT-FSK system is proposed as follows. The tone bandwidth is chosen as $150 \mathrm{MHz}$, with 16 centre frequencies so that the $2.5 \mathrm{GHz}$ EU UWB band is fully allocated. The repetition rate is selected to be $40 \mathrm{~ns}$ and based on the estimation for SER from section $2, K=4$ (the number of active tones) is chosen in order to satisfy the requirement of $500 \mathrm{MHz}$ bandwidth for UWB systems. This gives 1820 tone combinations. One symbol can therefore represent up to 10 bits.

In total, 7 measurements similar to the one described in section 4 has been performed for different distances between the transmitter and receiver. More details about these measurements can be found in [12]. The calibration was conducted for point $[1,1]$, then, the position was swept in a diagonal direction over all 40 available points so that the motion of the transmitter is modelled (displacement $\leq 33 \mathrm{~cm}$ ). The achieved data rate is $250 \mathrm{Mbps}$ and the number of transmitted bits for each position was $32 \cdot 10^{6}$. (note: that this will be reduced for multiple user access). In total 280 positions were tested using 7 calibration points, as presented in Fig. 10. 
For most of the positions the simulated BER yielded below $10^{-5}$. The worst case remained below $1 \cdot 10^{-2}$. Such BER can be corrected by a simple forward error correction algorithm. Also, the positions with higher BER error occurred for displacements larger than $15 \mathrm{~cm}$ from the calibration. In most practical cases new equalisation with a new pilot sequence will appear before the transmitter moves so far as mentioned in Section 4.

\section{Conclusions}

This paper has studied the practical deployment of Multi-tone FSK systems for UWB wireless communication. The main advantages of this scheme compared to commercial MB-OFDM systems are its low complexity and its ability to accommodate multiple users within the EU UWB band with bandwidth of $2.5 \mathrm{GHz}$ by using different tone subset codes to differentiate users.

Compared to impulse radio systems, MT-FSK system have higher duty cycle and can therefore achieve higher data-rates. In this paper, we used theoretical knowledge of the channel as well as empirical simulation to optimise a MT-FSK system. We find that signals with bandwidth between $150-500 \mathrm{MHz}$ are most suited for communication in an indoor channel. Such signals are shown to be an ideal combination of the advantages of impulse radio with those of UWB OFDM systems. The pulses are short enough to reduce the impact of frequency selective fading, whilst they remain long enough not to compromise duty cycle.

The spectral efficiency of the scheme is lower than in case of MB-OFDM, yet due to the wide available bandwidth, high data rates can be achieved as presented in the simulation using real channel impulse responses. A system employing MT-FSK with a data rate of up to 250 Mbps with BER below $10^{-5}$ proves that the concept of MT-FSK is a promising alternative to the standard approaches such as OFDM or impulse radio.

The proposed scheme relies on the use of energy detection which is a low complexity scheme avoiding the issues of WiMedia chips that have to perform highly complex FFT operations. Due to lower symbol repetition rate and analogue integration, it is also possible to use Digital-Analogue Processing at significantly lower clock rates than dictated by the UWB bandwidth. 
Therefore, MT-FSK is believed to be a viable candidate for high-speed UWB communication systems within the recently tightened regulatory limits on UWB communication systems. 


\section{References:}

[1] M.Z. Win; R. A. Scholtz; "Impulse radio: how it works," Communications Letters, IEEE , vol.2, no.2, pp.36-38, Feb 1998

[2] G. Heidari, WiMedia UWB - technology choice for wireless USB and Bluetooth. John Wiley \& Sons, 2008.

[3] Sipal, V.; Allen, B.; Edwards, D.; Honary, B; "20 Years of Ultrawideband: Opportunities and Challenges," IET Communications, accepted, in press, (special issue on UWB)

[4] Nakache, Y.P.; Orlik, P.; Gifford, W.M.; Molisch, A.F.; Ramachandran, I.; Fang, G.; Jinyun Zhang; , "Low-complexity ultrawideband transceiver with compatibility to multiband-OFDM," Ultra Wideband Systems, 2004. Joint with Conference on Ultrawideband Systems and Technologies. Joint UWBST \& IWUWBS. 2004 International Workshop on , vol., no., pp. 151- 155, 18-21 May 2004

[5] C. Luo; M. Medard; L. Zheng; , "On approaching wideband capacity using multitone FSK," Selected Areas in Communications, IEEE Journal on , vol.23, no.9, pp. 1830- 1838, Sept. 2005

[6] C. Luo; 'Communication for wideband fading channels : on theory and practice', PhD. Thesis, MIT, 2006

[7] R. Merit; "UWB startup Radiospire folds," EETimes www.eetimes.com, 21 April 2009

[8] Molisch, A. et.al. "IEEE 802.15.4a channel model - final report", technical report, www.ieee.org

[9] Sipal, V.; Gelabert, J.; Allen, B.; Stevens, C.; Edwards, D.; , "Frequency-selective fading of ultrawideband wireless channels in confined environments," Microwaves, Antennas \& Propagation, IET , vol.5, no.11, pp.1328-1335, August 192011

[10] Llano, G.; Reig, J.; Rubio, L.; , "Analytical Approach to Model the Fade Depth and the Fade Margin in UWB Channels," Vehicular Technology, IEEE Transactions on , vol.59, no.9, pp.4214-4221, Nov. 2010

[11] Sipal, V.; Allen, B.; Edwards, D.; , "Exploration of Nakagami fading in ultra-wideband wireless channels," Electronics Letters , vol.47, no.8, pp.520 -521, April 142011

[12] Sipal, V.; Allen, B.; Edwards, D.; , "Exploration and analysis of fade depth scaling," Antennas and Propagation Conference (LAPC), 2010 Loughborough , vol., no., pp.125-128, 8-9 Nov. 2010

[13] J. G. Proakis Digital Communications. $5^{\text {th }}$ Edition, McGraw Hill, 2008

[14] D'Amico, A.A.; Mengali, U.; Arias-de-Reyna, E.; , "Energy-Detection UWB Receivers with Multiple Energy Measurements," Wireless Communications, IEEE Transactions on , vol.6, no.7, pp.26522659, July 2007

[15] B. Allen et. al. Ultra Wideband Antennas and Propagation for Communications, Radar and Imaging. John Wiley \& Sons, 2006 


\section{List of Figures:}

Fig.1 Block diagram of transmitter and receiver using Multitone FSK

Fig. 2 Empirical cumulative distribution function of fading for signals at centre frequency of $7.25 \mathrm{GHz}$ for varying bandwidth

Fig. 3 Probability that signal received at a given distance will have power below the sensitivity of a state-of-the-art energy detector. The probability is shown in linear scale because for majority of "wideband data" points, the resulting probability is 0 , which cannot be expressed in logarithmic scale. This is given by the use of empiric CDF for fading.

Fig. 4 BER for $E\left\{\frac{E_{b}}{N_{0}}\right\}=10 \mathrm{~dB}$, when Nakagami fading is considered. Centre frequency $7.25 \mathrm{GHz}$

Fig. 5 The performance measure (3) of signal transmission for variable bandwidth

Fig. 6 Laboratory Plan

Fig. 7 Mean BER as function of tone bandwidth for measurements in 5 points spaced by $4 \mathrm{~cm}$. For tone bandwidth of $100 \mathrm{MHz}$, the BER was $7.1 \cdot 10^{-7}$

Fig. 8 Increase of BER with displacement over the 3dB-pulse-duration (highlighted by dashed vertical line) for several tone bandwidths (BW).

Fig. 9 BER versus symbol repetition period (change of duty cycle)

Fig. 10 BER performance of optimized Multitone Frequency Shift Keying system for 7 different position in rooms with transmitter movement $0-33 \mathrm{~cm}$. 
Figures:

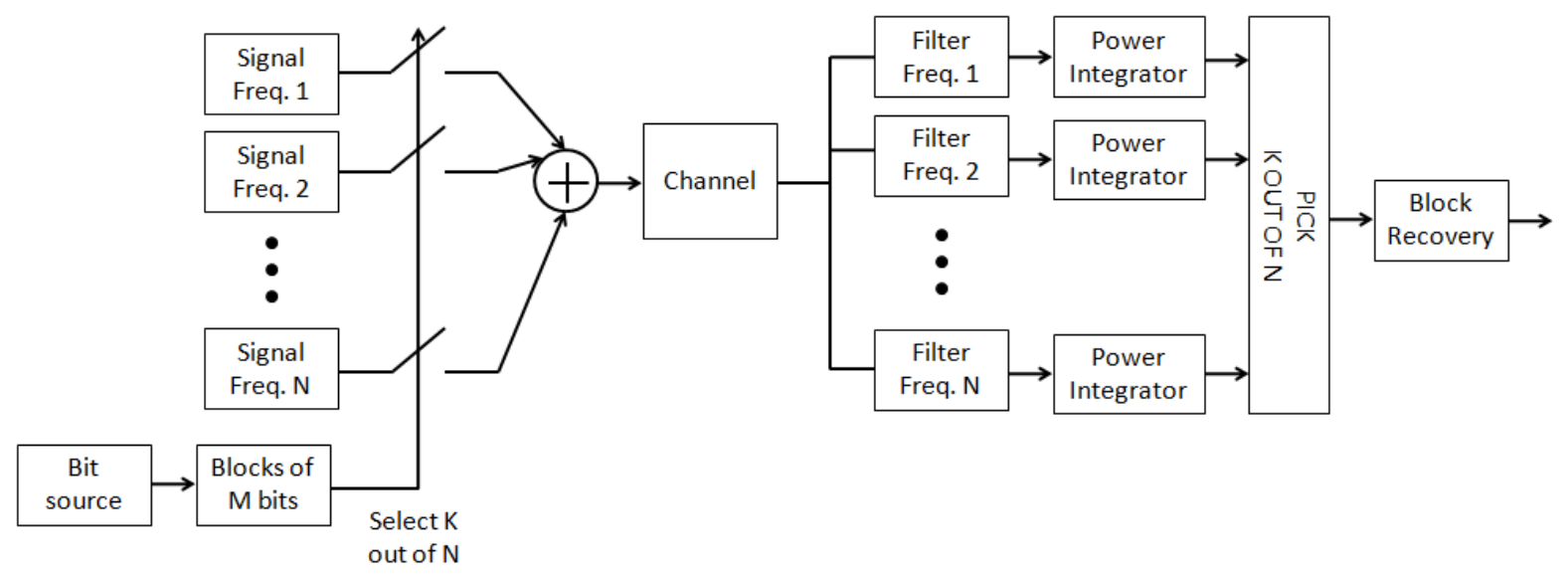

Fig.1 Block diagram of transmitter and receiver using Multitone FSK

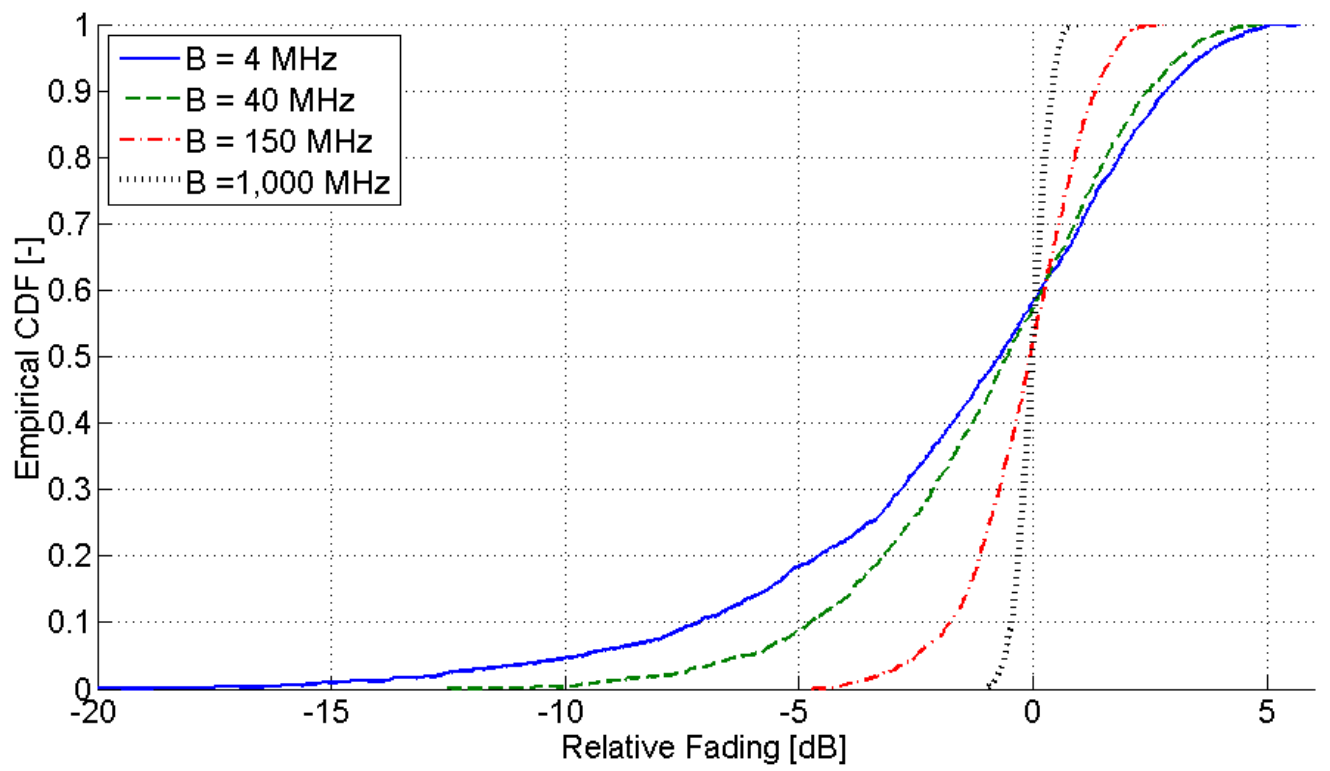

Fig. 2 Empirical cumulative distribution function of fading for signals at centre frequency of $7.25 \mathrm{GHz}$ for varying bandwidth 


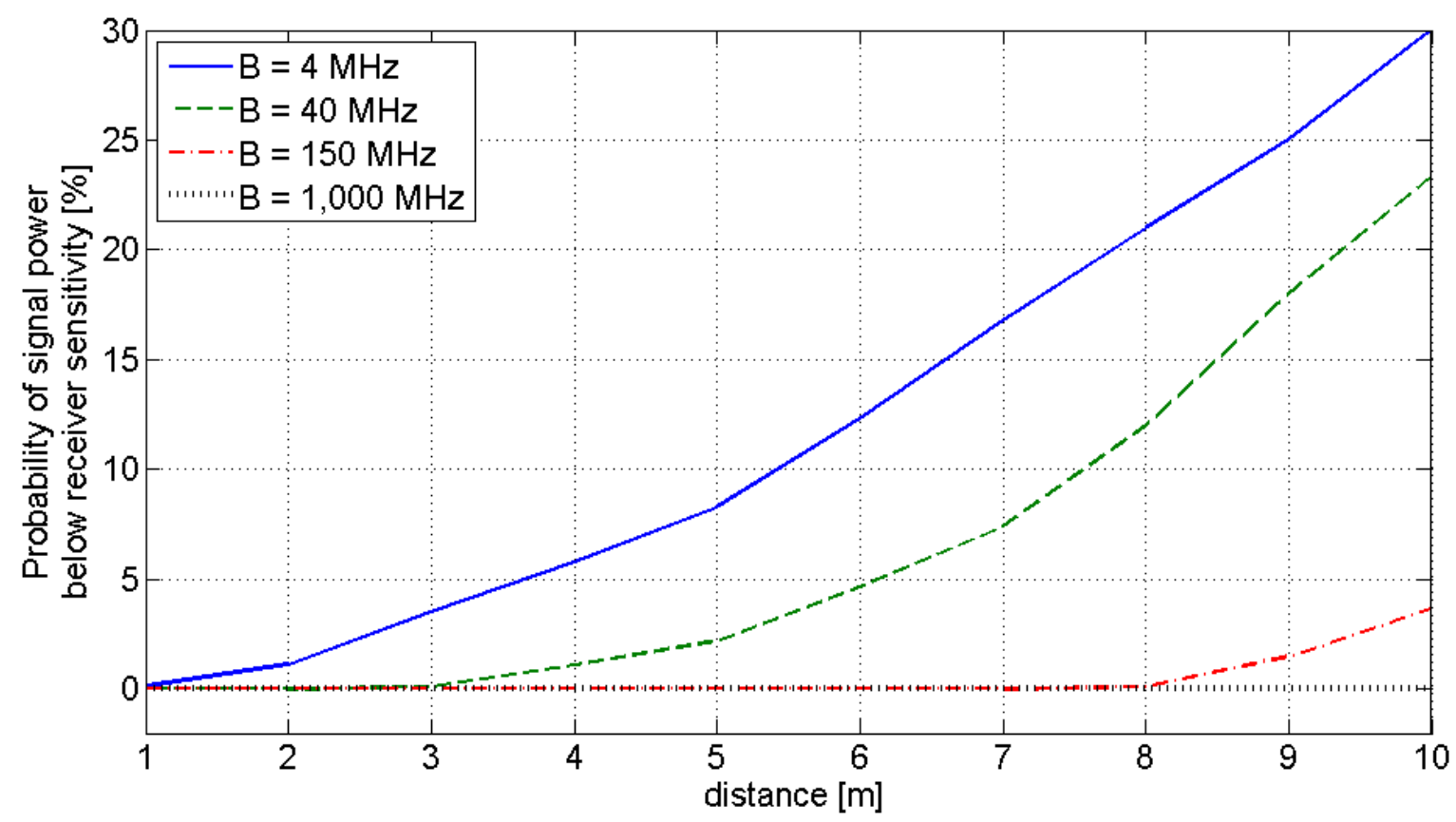

Fig. 3 Probability that signal received at a given distance will have power below the sensitivity of a state-of-the-art energy detector. The probability is shown in linear scale because for majority of "wideband data" points, the resulting probability is 0 , which cannot be expressed in logarithmic scale. This is given by the use of empiric CDF for fading.

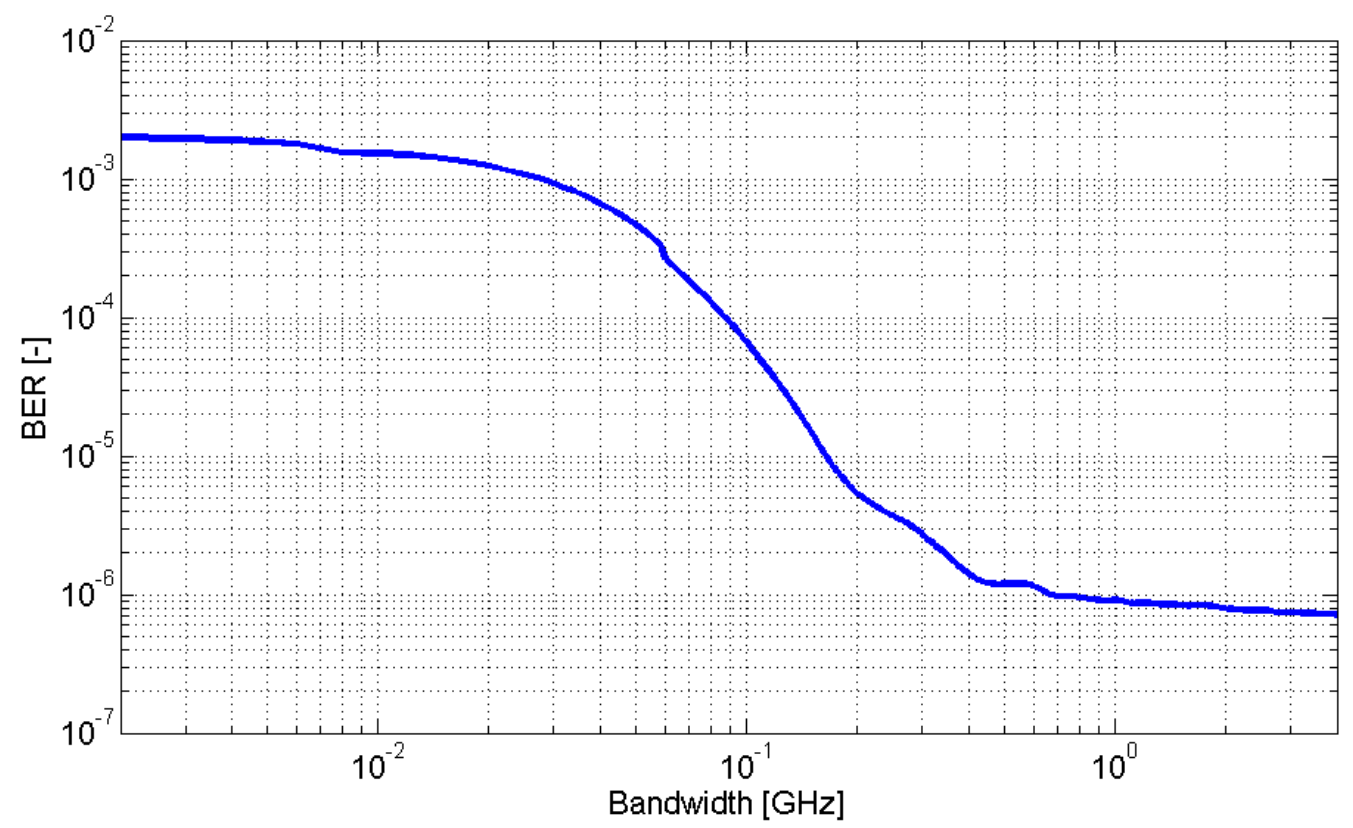

Fig. 4 BER for $E\left\{\frac{E_{b}}{N_{0}}\right\}=10 \mathrm{~dB}$, when Nakagami fading is considered. Centre frequency $7.25 \mathrm{GHz}$ 


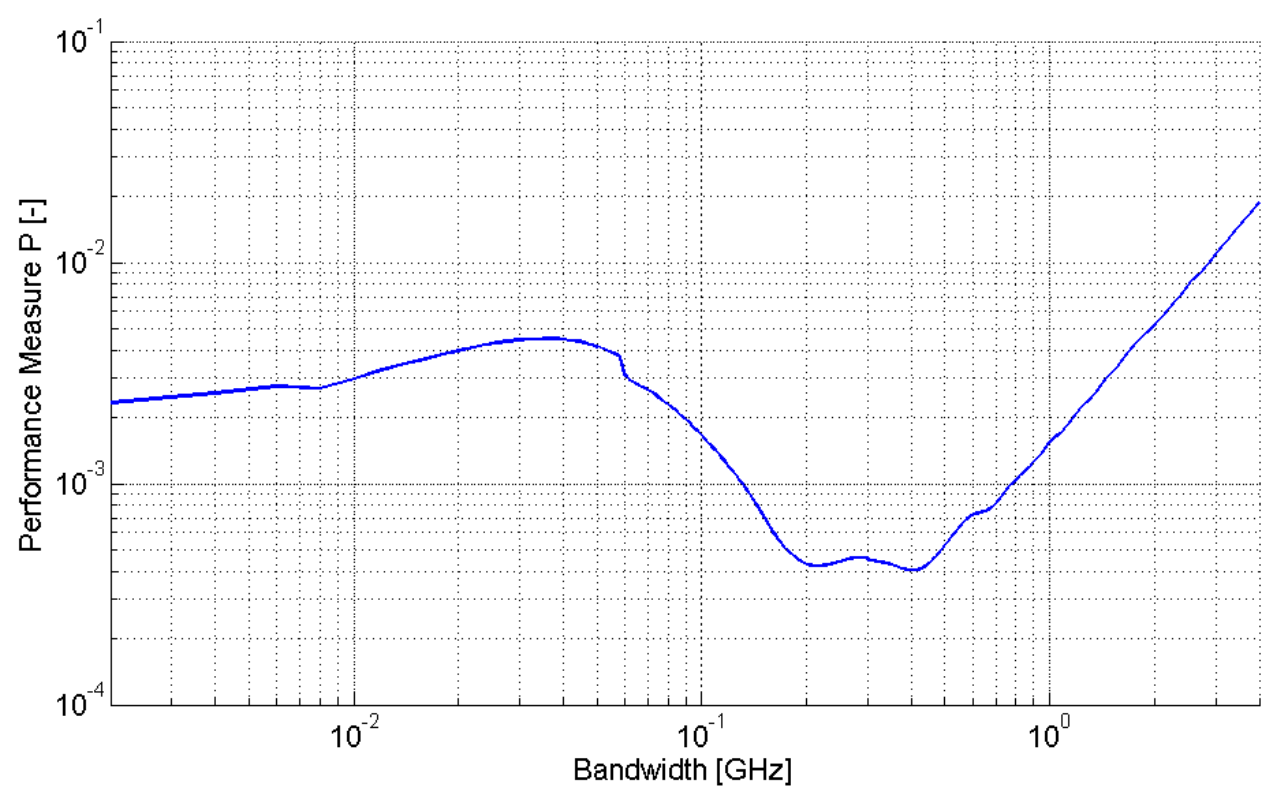

Fig.

Fig. 5 The performance measure (3) of signal transmission for variable bandwidth

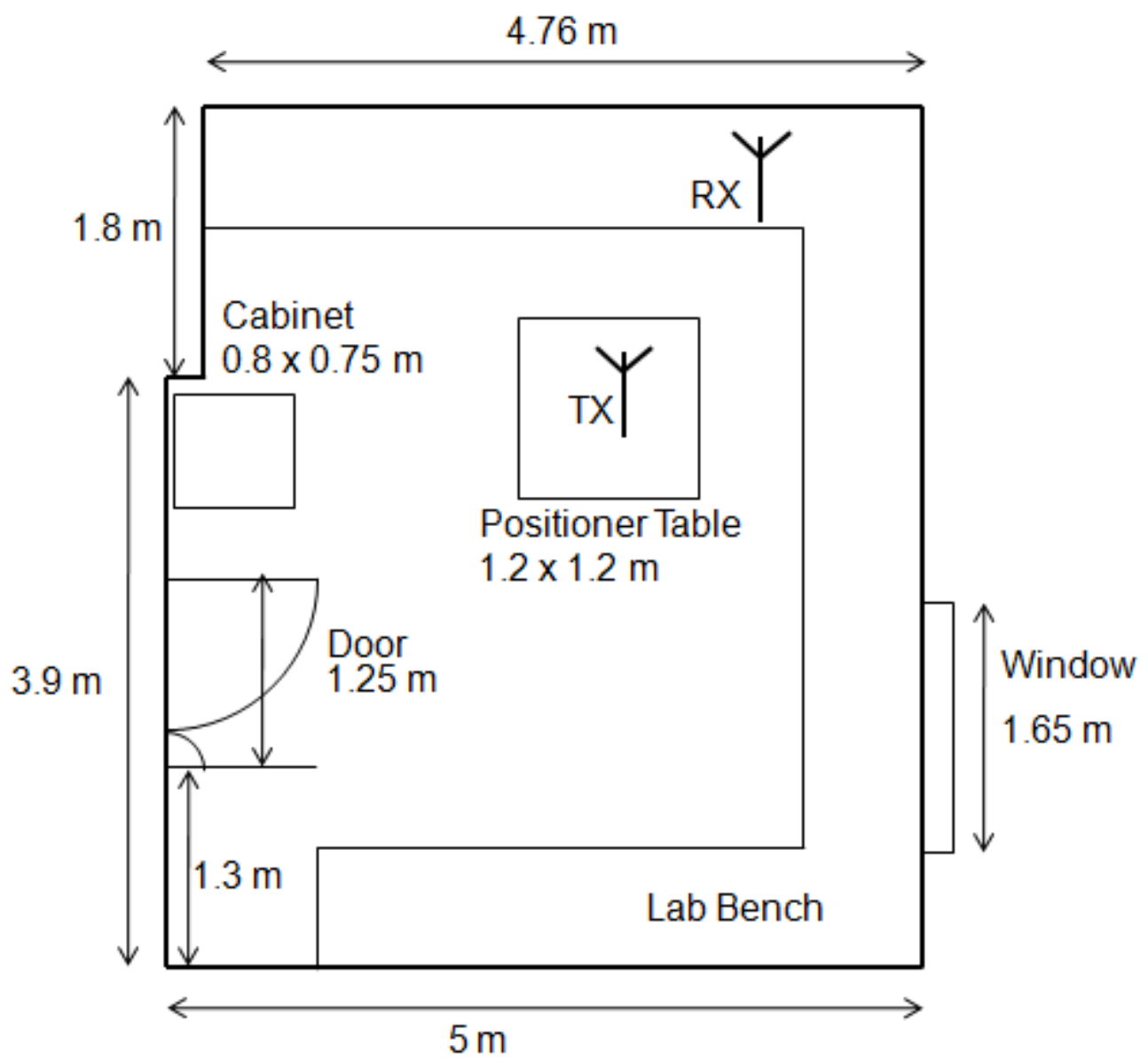

Fig. 6 Laboratory Plan 


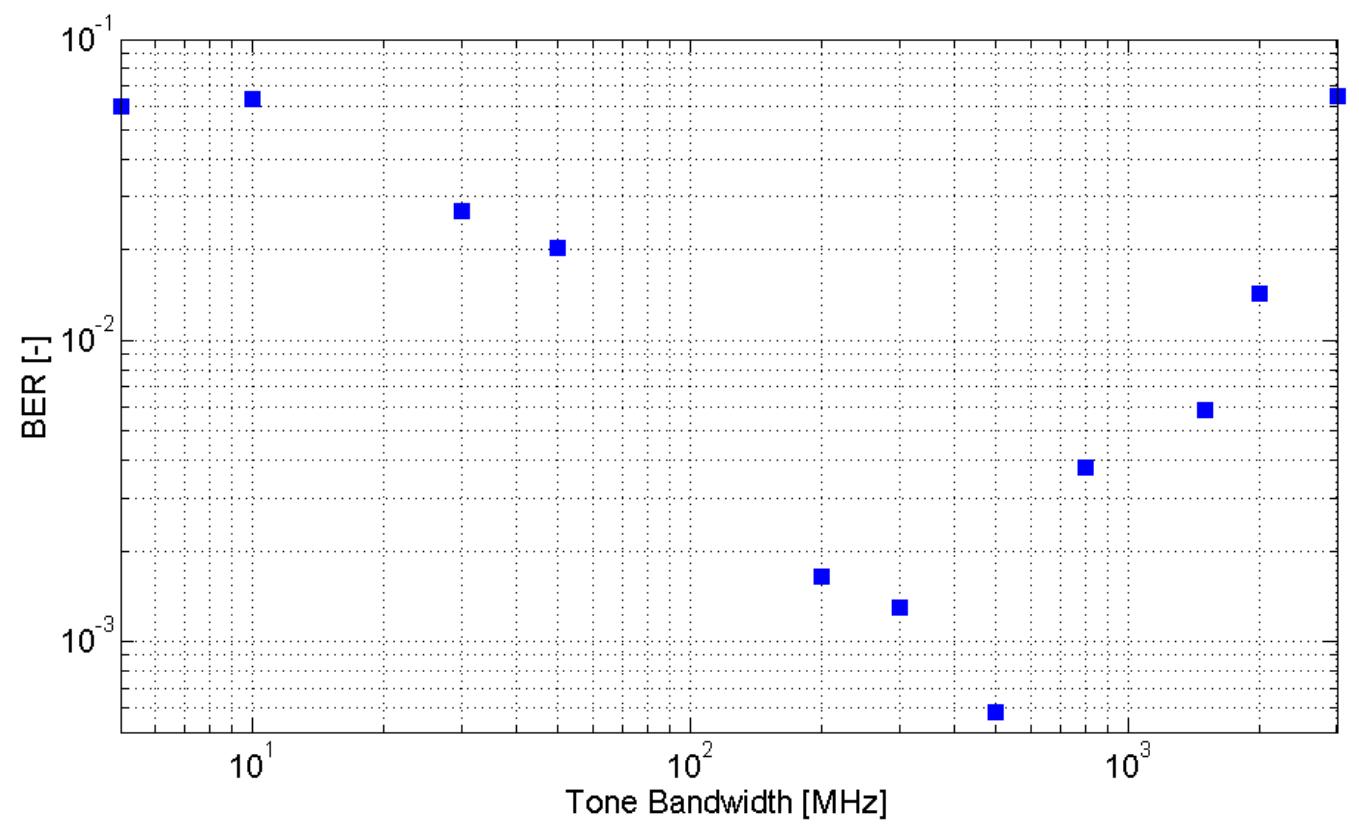

Fig. 7 Mean BER as function of tone bandwidth for measurements in 5 points spaced by $4 \mathrm{~cm}$. For tone bandwidth of $100 \mathrm{MHz}$, the BER was $7.1 \cdot 10^{-7}$

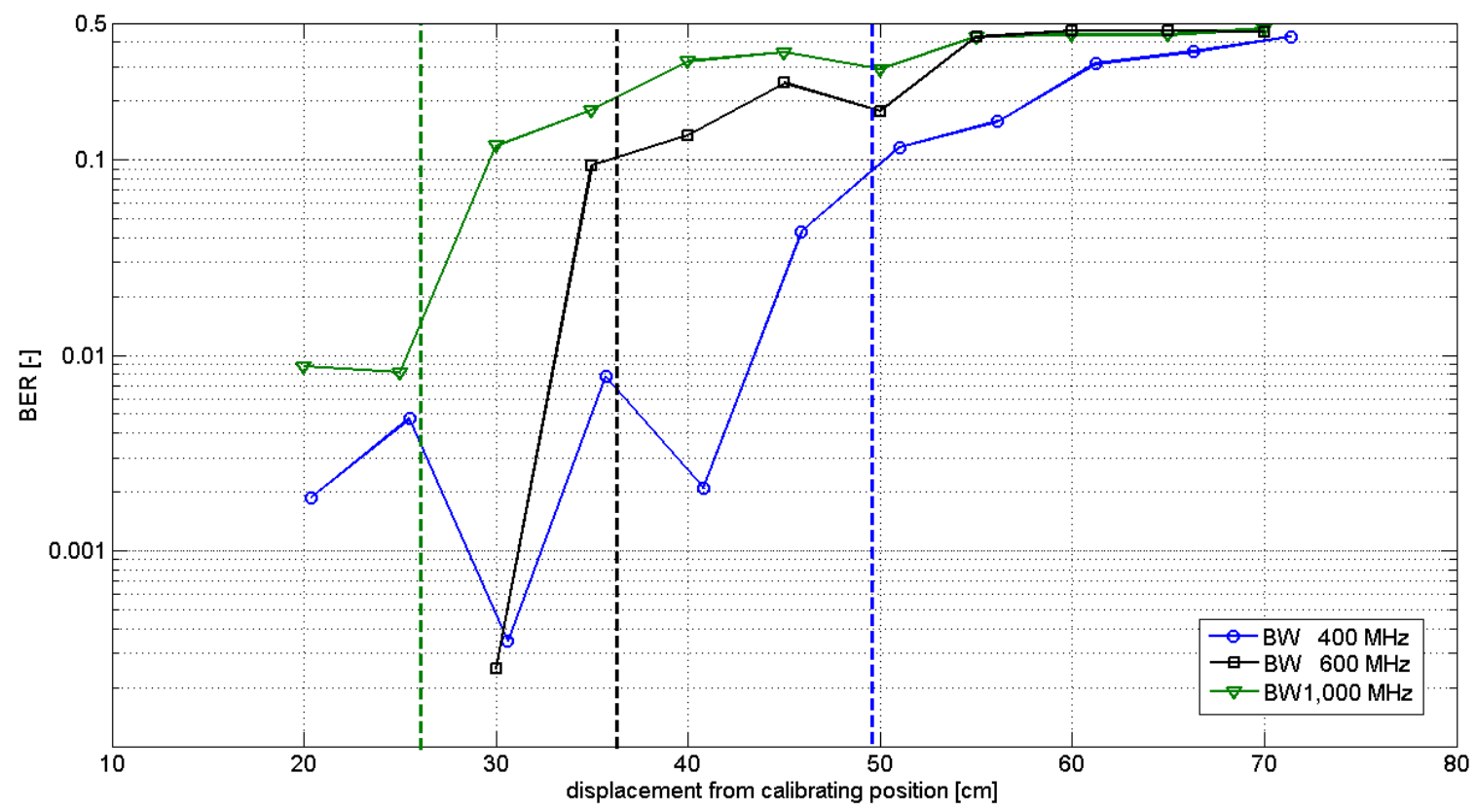

Fig. 8 Increase of BER with displacement over the 3dB-pulse-duration (highlighted by dashed vertical line) for several tone bandwidths (BW). 


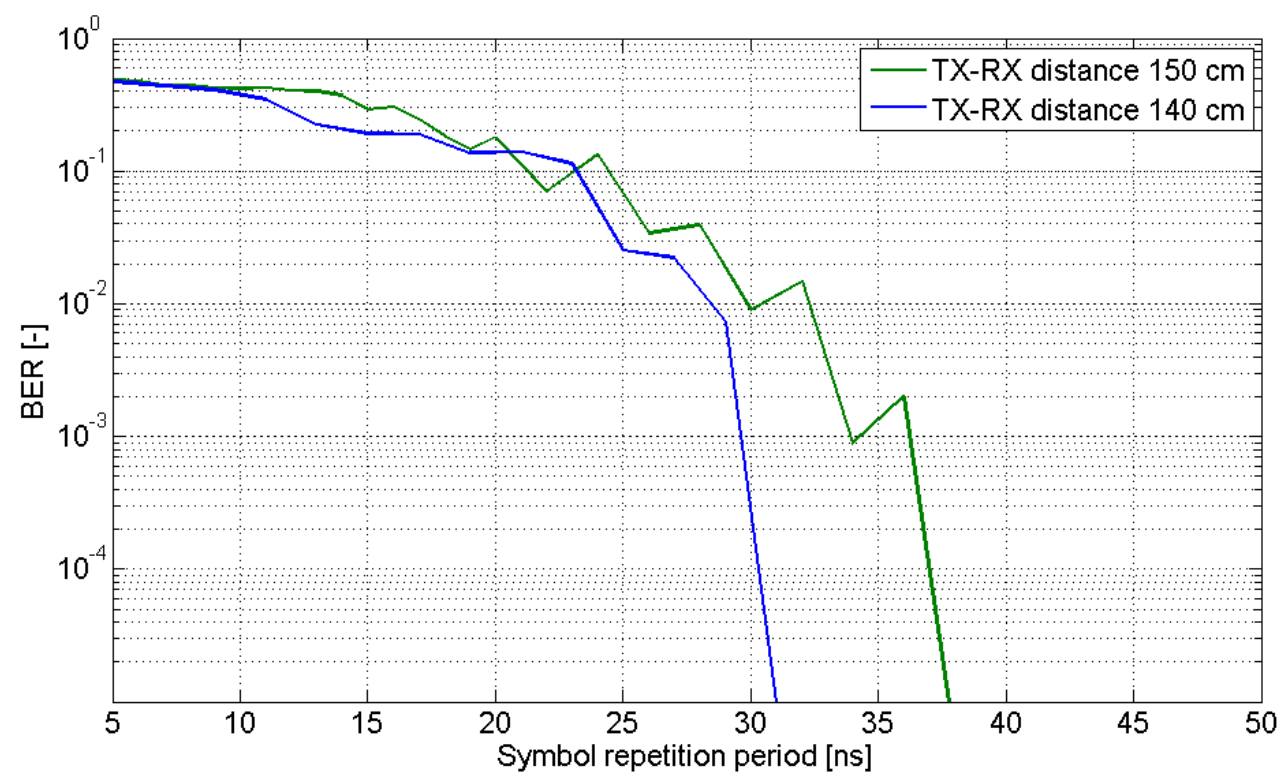

Fig. 9 BER versus symbol repetition period (change of duty cycle) showed for two TX-RX distances

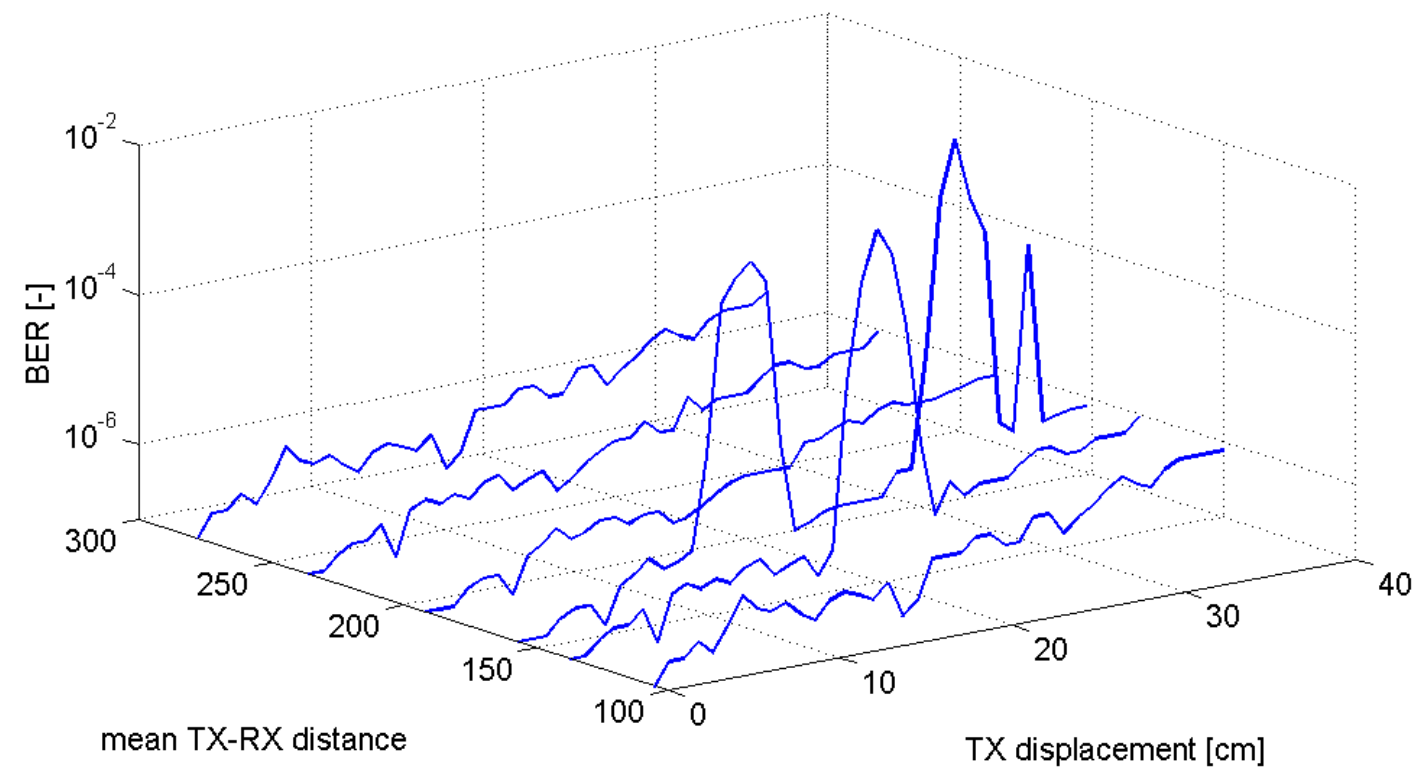

Fig. 10 BER performance of optimized Multitone Frequency Shift Keying system for 7 different position in rooms with transmitter movement $0-33 \mathrm{~cm}$. 